\title{
Proof of concept trial for a new theory-based intervention to promote child and adult behavior change
}

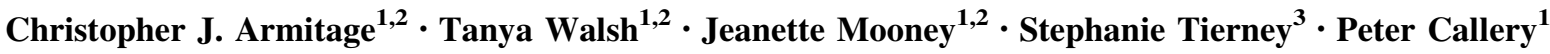

Received: February 4, 2019/Accepted: May 14, 2019/Published online: June 1, 2019

(C) The Author(s) 2019

\begin{abstract}
Interventions to change children's behavior typically target adults or children, but rarely both. The aims were to: (a) evaluate acceptability and feasibility of an innovative theory-based intervention designed to change both child and adult behavior, and (b) generate effect sizes for a definitive randomized controlled trial. The oral health of sixty children aged 5-9 years with a repaired cleft lip and/or palate was assessed before randomization to one of three conditions: (a) control group, (b) intervention group in which children and adults were asked to form implementation intentions, or (c) intervention plus booster group in which adults were additionally sent a reminder about the implementation intentions they and their children formed. Oral health assessments were repeated at 6-month followup alongside exit interviews. The procedures proved popular and participants exposed to the intervention additionally reported believing that forming implementation intentions was effective. Descriptive statistics generally showed oral health improvements across all conditions, although the effects were more marked in the intervention plus booster condition, where plaque improved by $44.53 \%$, gingivitis improved by $20.00 \%$ and free sugar consumption improved by $8.92 \%$ (vs. $6.43 \%$ improvement, $15.00 \%$ deterioration and $15.58 \%$ improvement in the control
\end{abstract}

Christopher J. Armitage

chris.armitage@manchester.ac.uk

1 Manchester Centre for Health Psychology, School of Health Sciences, Manchester Academic Health Science Centre, University of Manchester, Coupland Street, Oxford Road, Manchester M13 9PL, UK

2 Manchester University NHS Foundation Trust, Manchester, UK

3 University of Oxford, Oxford, UK group, respectively). Data collection procedures were acceptable and the intervention feasible. The effect sizes suggest that the intervention plus booster condition has sufficient promise to proceed to a fully-powered randomized controlled trial. The intervention has the potential to be adapted to tackle other child health behaviors and to be deployed at scale.

Keywords Implementation intention - Behavior change Randomized controlled trial · Cleft lip · Cleft palate . Children · Paediatrics · Feasibility · Parents · Carers · Oral health

\section{Introduction}

The leading causes of morbidity and mortality, such as cardiovascular disease and cancer, can be prevented through changes in key behaviors such as increasing physical activity, improving dietary intake, limiting alcohol consumption and stopping/not starting smoking (Global Burden of Disease Collaboration, 2015). Although the majority of interventions are designed to change the behaviors of adults, rather than children (e.g., Brunet et al., 2018), modifying the health behaviors of children is likely to yield the greatest health benefits. The few interventions designed to change children's behavior are typically targeted at adults (e.g., parents, teachers) or children (e.g., Nixon et al., 2012), but rarely both adults and children simultaneously. This is a potentially important omission because there are complex interactions between children and adults when it comes to managing health behavior change (e.g., Vallgarda et al., 2015), and models that suggest that targeting interpersonal processes may exert greater impact than targeting the individual alone (e.g., 
McLeroy et al., 1988). The aim of the present research was to test a theory-based behavior change intervention designed to promote both child and adult behavior change simultaneously.

It is clear that for many of the domains in which behavior change is important, the majority of people are already motivated to change (e.g., $68 \%$ of US smokers want to quit completely, Centers for Disease Control, 2017). This means that, in addition to considering ways to motivate people, researchers are paying increasing attention to behavior change interventions that will ensure people's existing good intentions are effectively translated into relevant behavior change. Accumulated research shows that implementation intentions, derived from Heckhausen and Gollwitzer's (1987) Rubicon model, are helpful in ensuring that good intentions are translated into action. Implementation intentions differ from other concepts found in models of behavior change (e.g., capabilities, opportunities, and motivations, see Michie et al., 2014) in so far as implementation intentions can be used to deliver multiple techniques (e.g., valued self-identities, Armitage et al., 2011; self-incentives, Brown et al., 2018) that bring about changes in behavior via changes in constructs such as capabilities, opportunities and motivations (Michie et al., 2014).

Implementation intentions (Gollwitzer, 1993) are 'ifthen' plans that involve linking critical cues (e.g., times of day, physical locations, internal sensations) with appropriate responses (e.g., self-talk, stimulus control). Specifying critical cues makes the 'if' part of the plan salient when it is encountered and the linked responses ('then') automatically spring to mind (Gollwitzer \& Sheeran, 2006). There is now a large body of work attesting to the effectiveness of implementation intention-based interventions, which typically exert medium-large $(d=0.65)$ effects on behavior change (e.g., Gollwitzer \& Sheeran, 2006). Despite the potential for implementation intentionbased interventions to change diverse behaviors, only a small number of previous studies deployed such interventions among children. Nevertheless, the signs are encouraging and implementation intention formation among children has been shown to help with: Impulse control (Gawrilow et al., 2011) preventing distractions in the classroom (Wieber et al., 2011), increasing fruit and vegetable intake (Gratton et al., 2007), preventing uptake of smoking (Conner \& Higgins, 2010), and increasing physical activity (Armitage \& Sprigg, 2010). Moreover, the effects are potentially long-lasting. For example, a study aimed at preventing smoking uptake in adolescents showed that the effects of an implementation intention-based intervention were sustained 2 years post-intervention (Conner \& Higgins, 2010). However, in each of these cases, implementation intentions were consistently targeted at children thereby ignoring the influence that adults exert on children's behavior. We thus developed an implementation intention-based intervention with the potential, where necessary, to promote both child and adult behavior change.

The context for the present study was oral health care among children, which is important because dental caries is the most prevalent of all diseases worldwide (Global Burden of Disease Collaboration, 2015). Guidelines to prevent dental caries highlight the importance of health behaviors that depend on a patient's input, particularly tooth-brushing as well as supervision for children (Department of Health and the British Association for the Study of Community Dentistry, 2009). Thus, maintenance of oral health care among children is an activity that should explicitly be the shared responsibility of children and adults. Development of the intervention is described elsewhere (Davies et al., 2017), but briefly, it involves a health care professional training both children and adults in the principles of implementation intentions and their formation with the help of a brief animation that was designed for the purposes of the present research. Following exposure to the animation, children and adults together form implementation intentions that they think will change their behavior (e.g., regular teethbrushing, avoiding sugary snacks).

Before proceeding to a full trial, we wanted to test proof of concept and so the aims of the present research were to: (a) test recruitment and retention rates, (b) assess the acceptability of the intervention and research procedures, and (c) generate effect sizes for a future definitive trial.

\section{Method}

\section{Participants and Procedure}

Participants were eligible if they were children aged 5-9 years with a repaired cleft lip and/or palate. Approximately 1 in 700 babies are affected by a cleft and have more plaque (Parapanisiou et al. 2009) and caries than the wider child population (Britton \& Welbury, 2010), making this a valuable population with which to test proof of concept. The exclusion criteria were: (a) having participated in intervention development work, (b) child allergic to food dyes, (c) unable to converse in English, and (d) families undergoing significant psychosocial difficulties (e.g. child protection, recent bereavement of a close family member), as identified by their cleft team.

Full ethical approval was obtained from the NHS Health Research Authority, Research Ethics Committee reference $15 / \mathrm{WM} / 0352$, and the trial was registered with the ISRCTN no: 45791053. Clinicians identified eligible participants in three cleft centers in the Midlands and North of 
England, and after receipt of written consent from parents/carers for them and their child to take part and verbal assent from the child, they were enrolled by a dental care practitioner based at each of the three cleft centers. Three dental care practitioners collected data in the child's home $(n=48,83 \%)$ or in a clinic $(n=10,17 \%)$ between July 2016 and October 2017.

\section{Design}

The design was a randomized proof of concept study. Children were allocated to one of three trial arms with a 1:1:1 allocation ratio using random permuted blocks. Allocation concealment was maintained through the use of sequentially numbered sealed opaque envelopes. The Clinical Trials Research Centre, University of Liverpool issued dental care practitioners with sequentially numbered envelopes containing randomly assigned allocations. After enrolling the participant onto the trial and taking baseline measurements the dental care practitioner opened an opaque envelope to reveal the allocation to control, intervention, or intervention plus booster group. Questionnaires and clinical assessments were repeated and an exit interview recorded at 6 months follow-up. It only proved feasible for the follow up visits to be conducted by the dental care practitioner who conducted the first visit and so blinding was not possible.

\section{Materials}

All participants received standard dental care education, which included recommendations about which toothpaste to use, information about the consequences of consuming sugary snacks/drinks, and tooth brushing techniques. Those randomized to the two intervention groups watched a 3-min animation (https://www.youtube.com/watch?v= UCX1z_gJjqM\&feature=youtu.be) that explained the principles behind 'if-then' plans and provided examples of what might make effective 'if-then' plans, including plans to brush one's teeth and avoid sugary snacks. Following the animation, children and adults received a laminated sheet on which to write their implementation intention. The dental care practitioners photographed the laminated sheets, which participants retained. Repeated administration of implementation intentions have previously boosted the effects of the intervention (Chapman \& Armitage, 2010) and so participants in the intervention plus booster condition were contacted via mail, SMS or e-mail depending on parent/carer preference three months postbaseline to remind them of their implementation intention.

\section{Measures}

At baseline only, demographic data about child's gender, age and level of deprivation, using the Indices of Multiple Deprivation (http://dclgapps.communities.gov.uk/imd/ idmap.html) were assessed. All other measures were taken at baseline and six-month follow-up.

\section{Oral health status}

Dental care practitioners conducted visual inspections of plaque and gingivitis, which are indicators for risk of dental caries, at baseline and six-month follow-up. The Plaque Control Record (O'Leary et al., 1972) records the presence of plaque on individual tooth surfaces and is calculated by dividing the number of surfaces with plaque by the number of total tooth surfaces and multiplying by 100. The Modified Gingival Index (Lobene et al., 1986) has been shown to correlate well with bleeding indices (Lobene et al., 1986) and has been widely employed in clinical trials. The index uses a visual assessment of the level of inflammation present on both papillary and marginal gingival tissues on a scale of $0-4$. An overall gingivitis score can then be ascribed.

\section{Dietary intake}

Consumption of free sugars was assessed using the Day in the Life questionnaire, which asks participants to recall food intake over a $24 \mathrm{~h}$ period (Edmunds \& Ziebland, 2002); it was developed in the UK to assess the dietary intake of children aged 7-9. The Day in the Life questionnaire enables researchers to identify the number of times children had free sugars in food or drinks in the previous $24 \mathrm{~h}$.

\section{Oral health behaviors}

Frequency of toothbrushing, visits to dentists and use of fluoride were assessed using items selected from the Children's Dental Health Survey (Pendry et al., 2004).

\section{Sample size}

A power calculation was not conducted because this was not a definitive trial. Julious (2005) recommends that pilot trials should aim to recruit at least 12 participants per group in order to obtain meaningful data. We estimated that data from 20 children and their parent(s)/carer(s) in each arm would provide sufficient information for the purposes of establishing acceptability, feasibility and calculating effect sizes. 


\section{Data analysis}

Formal hypothesis testing for effectiveness was not undertaken as the aim of this proof of concept trial was not to assess effectiveness and as such the study is underpowered for this purpose. It was not our intention to compare treatment groups and we did not test hypotheses. Cumulative and monthly (to assess season variation) recruitment figures were calculated, as was loss to follow up. Descriptive analyses were undertaken to identify the total number of potentially eligible participants and the number and proportion of participants who enrolled onto the trial. The completeness and variability of clinical and patient-reported outcomes was assessed using simple descriptive statistics involving calculation of ranges, frequency distributions and measures of central tendency and dispersion.

\section{Enrolment}

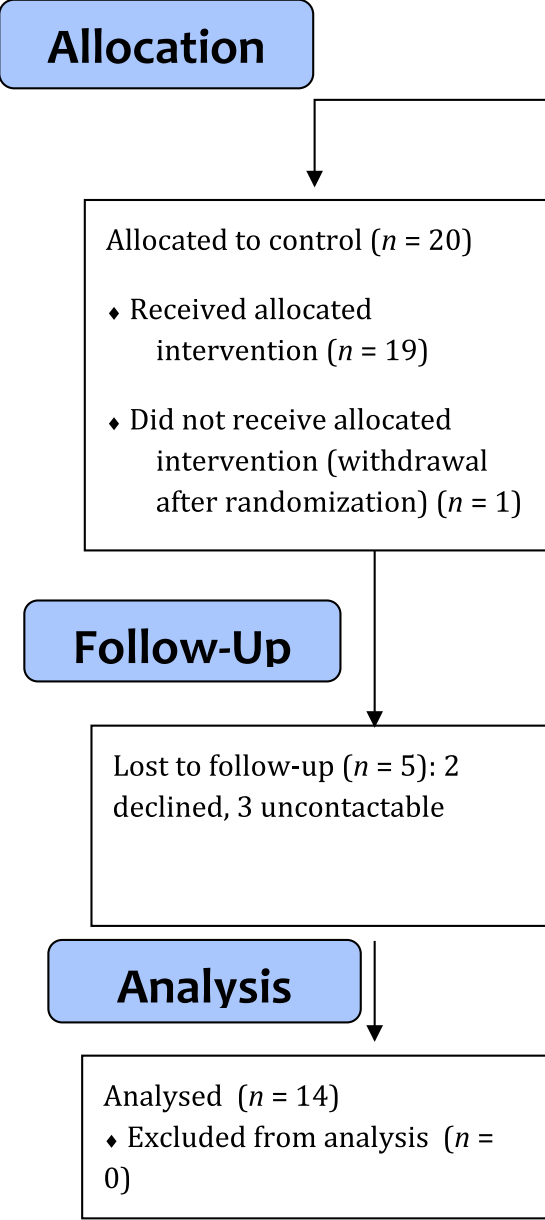

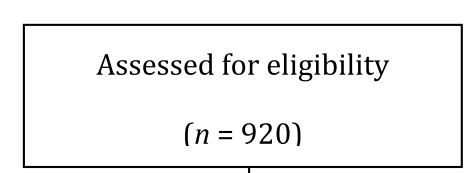

Allocated to intervention $(n=$ 20)

- Received allocated intervention ( $n=19)$

- Did not receive allocated intervention (withdrawal after randomization) ( $n=1)$

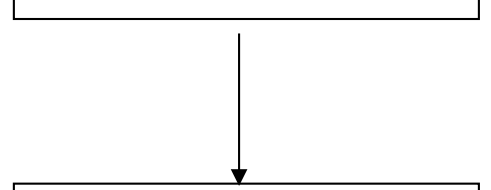

Lost to follow up ( $n=3)$ : 1 declined, 2 uncontactable,

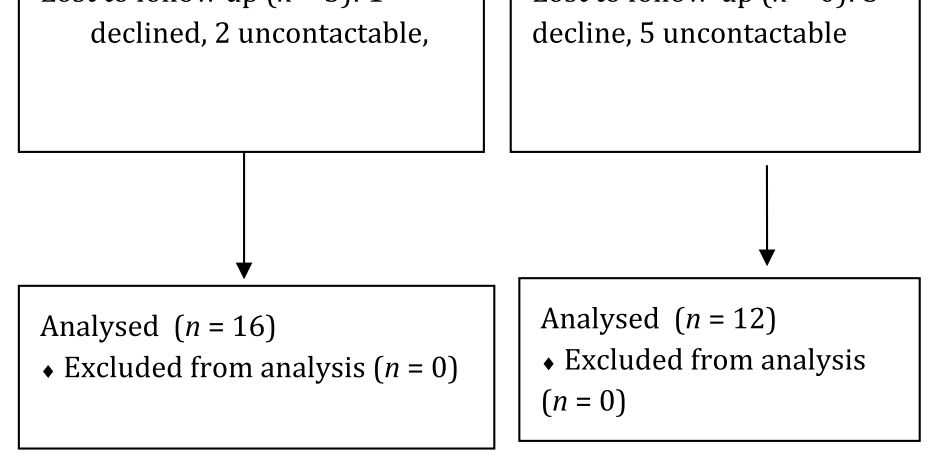

Excluded $(n=860)$

- Not meeting inclusion criteria (not age 5-9 years $=690$; insufficient English language = 18; Psychosocial difficulties $=19$. Total $\mathrm{n}=727$ )

- Declined to participate $(n=133)$

Fig. 1 CONSORT flow diagram 


\section{Results}

\section{Feasibility}

Nine hundred and twenty participants were assessed for eligibility, and 860 were excluded, largely on the grounds that the child did not meet the 5-9 years inclusion criterion (Fig. 1). Nevertheless, 60 children were recruited into the study over the period June 2016 to March 2017 and 20 children randomly allocated to each of the control, intervention and intervention plus booster conditions. Fiftyeight children completed baseline assessments, and 50 children provided follow-up data. Although the sample sizes are small, the control and intervention groups were broadly comparable in terms of key demographic variables, including gender, age, deprivation and clinical variables (Table 1).

\section{Acceptability}

\section{Procedures}

Forty-one adults completed exit interviews and none of the participants reported any concerns about the study, indicating the acceptability of the procedures. All adults, including people in the control as well as both intervention groups, reported that looking after their child's teeth improved during the study.

\section{Intervention}

Dental care practitioners reported that it took them between 5 and $25 \mathrm{~min}$ (median $=15 \mathrm{~min}$ ) to deliver the intervention and for children and/or adults to form implementation intentions. Children and adults mostly formed implementation intentions together, $n=25(64 \%)$, although $9(23 \%)$ children and $5(13 \%)$ parents formed implementation intentions alone. The content of the implementation intentions included: ensuring that teeth are brushed as part of a daily routine, teeth brushing for the recommended
2 min, and identifying substitutes for drinks and snacks containing free sugars. On $79.5 \%$ of occasions $(n=31)$, dental care practitioners reported that children and adults described the process of forming implementation intentions as "easy" or "very easy". Twenty six (of 29, 89.6\%) adults whose children were allocated to one of the intervention groups described implementation intentions as "an effective strategy".

\section{Main outcomes}

Baseline and 6-month follow-up data on clinical outcomes are presented in Table 2. Observed plaque improved in all three conditions, with the most marked improvement for the intervention plus booster condition, in which plaque reduced by $44.53 \%$ compared with $24.16 \%$ in the next-best (intervention) condition. A similar pattern emerged for observed gingivitis, which improved in the intervention plus booster condition (20.00\%), but deteriorated in both the control condition (by 15.00\%) and the intervention condition (by $55.55 \%$ ). Free sugar consumption did not change in the intervention condition, but was improved in both the control $(15.58 \%)$ and intervention plus booster $(8.92 \%)$ conditions.

\section{Sample size calculation for a powered trial}

A sample size calculation for a fully-powered trial was computed based on the Plaque Control Record data collected during the present study. The parameters were: Plaque Control Record value in control group of 50, smallest observed absolute reduction in Plaque Control Record of 10, observed standard deviations, power of $80 \%$ and significance level of 0.01 to account for the multiple pairwise testing in a three arm trial. A definitive trial would therefore require 204 participants per arm, a total sample size of 612 .

Table 1 Baseline characteristics of the sample

\begin{tabular}{llll}
\hline Variables & Control, $n=19$ & Intervention-only, $n=19$ & Intervention plus booster, $n=20$ \\
\hline Age, $M(S D)$ & $7.96(1.46)$ & $7.23(1.50)$ & $7.69(1.67)$ \\
Female child, $n(\%)$ & $11(58)$ & $7(37)$ & $8(40)$ \\
Two most-deprived quintiles, $n(\%)$ & $9(45)$ & $6(30)$ & $8(40)$ \\
Seen at home, $n(\%)$ & $17(90)$ & $16(84)$ & $15(75)$ \\
Provided follow-up data, $n(\%)$ & $14(74)$ & $16(84)$ & $20(70)$ \\
Plaque control record, $M(S D)$ & $53.69(32.43)$ & $54.98(27.84)$ & $53.85(26.11)$ \\
Modified gingivitis index, $M(S D)$ & $0.25(0.29)$ & $0.13(0.20)$ & $0.16(0.26)$ \\
Free sugar consumption, $M(S D)$ & $3.05(1.13)$ & $3.53(1.31)$ & $3.25(1.16)$
\end{tabular}


Table 2 Baseline and follow-up clinical characteristics of the sample completing the trial

\begin{tabular}{|c|c|c|c|c|c|c|}
\hline \multirow[t]{2}{*}{ Dependent variables } & \multicolumn{2}{|c|}{ Baseline } & \multicolumn{2}{|c|}{ Follow-up } & \multirow{2}{*}{$\begin{array}{l}M \text { difference } \\
(S D)\end{array}$} & \multirow{2}{*}{$\begin{array}{l}\% \text { Change } \\
\text { From baseline }\end{array}$} \\
\hline & $M$ & $S D$ & $M$ & $S D$ & & \\
\hline \multicolumn{7}{|l|}{ Plaque control record } \\
\hline Control, $n=14$ & 53.33 & 34.21 & 49.90 & 36.10 & $-3.43(28.89)$ & -6.43 \\
\hline Intervention, $n=16$ & 51.86 & 28.85 & 39.33 & 28.90 & $-12.53(25.60)$ & -24.16 \\
\hline Intervention plus booster, $n=12$ & 52.41 & 28.62 & 29.07 & 26.67 & $-23.34(30.11)$ & -44.53 \\
\hline \multicolumn{7}{|l|}{ Modified gingivitis index } \\
\hline Control, $n=14$ & 0.20 & 0.24 & 0.23 & 0.39 & $+0.03(0.26)$ & +15.00 \\
\hline Intervention, $n=16$ & 0.09 & 0.14 & 0.14 & 0.22 & $+0.05(0.18)$ & +55.55 \\
\hline Intervention plus booster, $n=12$ & 0.10 & 0.22 & 0.08 & 0.15 & $-0.02(0.26)$ & -20.00 \\
\hline \multicolumn{7}{|l|}{ Free sugar consumption } \\
\hline Control, $n=14$ & 3.21 & 1.25 & 2.71 & 1.64 & $-0.50(1.91)$ & -15.58 \\
\hline Intervention, $n=16$ & 3.69 & 1.35 & 3.69 & 1.58 & $0(1.26)$ & 0 \\
\hline Intervention plus booster, $n=12$ & 3.14 & 1.29 & 2.86 & 1.75 & $-0.29(1.98)$ & -8.92 \\
\hline
\end{tabular}

\section{Discussion}

This is the first test of a theory-based behavior change intervention designed explicitly to change both child and adult behavior simultaneously. The study demonstrated that children and adults can be recruited and complete a randomized trial, find the intervention and methods of assessment acceptable, and view the intervention as useful. Thus, the present study demonstrated the feasibility of both the intervention and data collection procedures. Although not designed to test effectiveness, the clinical assessments were consistent with reductions in plaque in the intervention groups, with an increased effect with use of a booster. Together with the size of the effects observed, the present study suggests that it would be useful to proceed to a definitive randomized controlled trial. If proved effective the brief intervention could be integrated into routine practice within the cleft population and has substantial potential for adaptation to address other child and adult health behaviors.

The present research contributes to a small evidencebase showing that implementation intentions can be effective as the basis for interventions designed to change children's behavior (e.g., Armitage \& Sprigg, 2010). This is important because major public health gains will only be achieved with greater emphasis on prevention/intervention in childhood. Moreover, the present means by which people formed implementation intentions (i.e., by watching a video animation) was novel and contributes to an evidence base showing that as long as the "if-then" format is followed, the means by which implementation intentions are delivered can be flexible. Thus, the present research contributes to a literature showing that effective implementa- tion intentions can be self-generated or researcher-provided (Armitage, 2009), administered online (Armitage, 2015) or even placed on the side of wine bottles (Armitage \& Arden, 2016), by encouraging children and adults to form implementation intentions by means of an animation. This means that the intervention has the potential to be deployed at scale with high public health reach.

\section{Limitations}

Although the present research takes the literature on implementation intentions forward in some important respects, there are potential limitations. First, while the use of home visits for data collection and intervention delivery was acceptable and feasible it may not be a practical model for a larger study. However, a minority of assessments and interventions $(n=10,17 \%)$ were completed in clinics. Given that there seemed to be no difference in acceptability between home visits versus clinic administration, it might be the case that delivering the intervention in clinics alongside routine check-ups is more cost-effective than home visits. Provision within the clinics would also provide opportunities for families unable to converse in English to receive the intervention with interpreters on hand to facilitate routine consultations.

A second limitation is that although dental care practitioners were blinded to the initial clinical assessments, the distances of homes from cleft centers meant that it was not possible to send a blinded assessor to the 6-month followup visits. This lack of blinding at follow-up raises the possibility of bias in the clinical assessments. However, follow-up assessments of gingivitis in the intervention condition produced a notable increase in gingivitis 
$(+7.69 \%)$ at the same time as a marked decrease in plaque $(-28.46 \%)$, which might suggest some level of confidence in the objectivity of the assessments. Nevertheless, it would be preferable to ensure that all clinical assessments are blinded, which might be achievable in a clinic-based setting.

Third, implementation intentions operate by increasing the salience of critical cues and automatizing cue-response links (Gollwitzer \& Sheeran, 2006). As yet, there is no agreed way of directly measuring this deployment of strategic automaticity outside the laboratory, meaning there was no way of assessing process, which is a further potential limitation.

\section{Conclusions}

This study demonstrates the appropriateness, feasibility and potential of an implementation intention-based intervention to promote oral health in children with a repaired cleft, with potential for wider application in child health.

Acknowledgements The NHS Health Research Authority, Research Ethics Committee reference 15/WM/0352, approved the study. The trial was registered with the ISRCTN No.: 45791053. This paper presents independent research funded by the National Institute for Health Research (NIHR) under its Research for Patient Benefit (RfPB) Programme (Grant Reference Number PB-PG-0613-31022). The NIHR Manchester Biomedical Research Centre and NIHR Greater Manchester Patient Safety Translational Research Centre also supported the research. The views expressed are those of the author(s) and not necessarily those of the NHS, the NIHR or the Department of Health and Social Care. We would like to thank dental care practitioners Jessica Butcher, Caroline Connolly and Laura Stannage who recruited participants and collected the data as well as Saff Bahm, Haydn Bellardie, Vicky Brand, Nancy Bray, Victoria Clark, Karen Davies, Susana Dominquez-Gonzalez, Lars Enocson, Kat Kandiah, Yin-Ling Lin, Deborah Moore, James Munro, Kevin O'Brien, Bill Shaw, Martin Tickle, and Cath Wright who advised on the development of the intervention and the study.

\section{Compliance with ethical standards}

Conflict of interest Christopher J. Armitage, Tanya Walsh, Jeanette Mooney, Stephanie Tierney, and Peter Callery declare that they have no conflict of interest.

Human and animal rights and Informed consent All procedures followed were in accordance with ethical standards of the responsible committee on human experimentation (institutional and national) and with the Helsinki Declaration of 1975, as revised in 2000. Informed consent was obtained from all patients for being included in the study.

Open Access This article is distributed under the terms of the Creative Commons Attribution 4.0 International License (http:// creativecommons.org/licenses/by/4.0/), which permits unrestricted use, distribution, and reproduction in any medium, provided you give appropriate credit to the original author(s) and the source, provide a link to the Creative Commons license, and indicate if changes were made.

\section{References}

Armitage, C. J. (2009). Effectiveness of experimenter-provided and self-generated implementation intentions to reduce alcohol consumption in a sample of the general population: A randomized exploratory trial. Health Psychology, 28, 545-553.

Armitage, C. J. (2015). Randomised test of a brief psychological intervention to reduce and prevent emotional eating in a community sample. Journal of Public Health, 37, 438-444. https://doi.org/10.1093/pubmed/fdv054

Armitage, C. J., \& Arden, M. A. (2016). Enhancing the effectiveness of alcohol warning labels with a self-affirming implementation intention. Health Psychology, 35, 1159-1163. https://doi.org/10. 1037/hea0000376

Armitage, C. J., Harris, P. R., \& Arden, M. A. (2011). Evidence that self-affirmation reduces alcohol consumption: Randomized exploratory trial with a new, brief means of self-affirming. Health Psychology, 30, 633-641.

Armitage, C. J., \& Sprigg, C. A. (2010). The roles of behavioral and implementation intentions in changing physical activity in young children with low socioeconomic status. Journal of Sport and Exercise Psychology, 32, 359-376.

Britton, K. F., \& Welbury, R. R. (2010). Dental caries prevalence in children with cleft lip/palate aged between 6 months and 6 years in the West of Scotland. European Archives of Paediatric Dentistry, 11, 236-241.

Brown, E. M., Smith, D. M., \& Armitage, C. J. (2018). Self-incentives uniquely boost cessation in community-based stop smoking programs: Randomized controlled trial. Annals of Behavioral Medicine. https://doi.org/10.1093/abm/kay056

Brunet, J., Wurz, A., \& Shallwani, S. M. (2018). A scoping review of studies exploring physical activity among adolescents and young adults diagnosed with cancer. Psycho-Oncology, 27, 1875-1888. https://doi.org/10.1002/pon.4743

Centers for Disease Control and Prevention. (2017). Quitting smoking among adults-United States, 2000-2015. Morbidity and Mortality Weekly Report, 65, 1457-1464.

Chapman, J., \& Armitage, C. J. (2010). Evidence that boosters augment the long-term impact of implementation intentions on fruit and vegetable intake. Psychology and Health, 25, 365-381.

Conner, M., \& Higgins, A. R. (2010). Long-term effects of implementation intentions on prevention of smoking uptake among adolescents: A cluster randomized controlled trial. Health Psychology, 29, 529-538. https://doi.org/10.1037/a0020317

Davies, K., Armitage, C. J., Lin, Y.-L., Munro, J., Walsh, T., \& Callery, P. (2017). Development of an implementation intentionbased intervention to change children's and parent-carers' behaviour. BMC Pilot and Feasibility Studies, 4, 20. https:// doi.org/10.1186/s40814-017-0171-6

Department of Health and the British Association for the Study of Community Dentistry. (2009). Delivering better oral health: An evidence-based toolkit for prevention. London: HMSO.

Edmunds, L. D., \& Ziebland, S. (2002). Development and validation of the Day in the Life Questionnaire (DILQ) as a measure of fruit and vegetable questionnaire for 7-9 year olds. Health Education Research, 17, 211-220. https://doi.org/10.1093/her/17.2.211

Gawrilow, C., Gollwitzer, P. M., \& Oettingen, G. (2011). If-then plans benefit executive functions in children with ADHD. Journal of Social and Clinical Psychology, 30, 616-646.

Global Burden of Disease Study 2013 Collaborators. (2015). Global, regional, and national incidence, prevalence, and years lived with disability for 301 acute and chronic diseases and injuries in 188 countries, 1990-2013: A systematic analysis for the Global Burden of Disease Study 2013. Lancet, 386, 743-800. https:// doi.org/10.1016/S0140-6736(15)60692-4 
Gollwitzer, P. M. (1993). Goal achievement: The role of intentions. European Review of Social Psychology, 4, 141-185.

Gollwitzer, P. M., \& Sheeran, P. (2006). Implementation intentions and goal achievement. Advances in Experimental Social Psychology, 38, 69-119. https://doi.org/10.1013/S0065-2601

Gratton, L., Povey, R., \& Clark-Carter, D. (2007). Promoting children's fruit and vegetable consumption: Interventions using the theory of planned behaviour as a framework. British Journal of Health Psychology, 12, 639-650. https://doi.org/10.1348/ 135910706X171504

Heckhausen, H., \& Gollwitzer, P. M. (1987). Thought contents and cognitive functioning in motivational versus volitional states of mind. Motivation and Emotion, 11, 101-120.

Julious, S. A. (2005). Sample size of 12 per group rule of thumb for a pilot study. Pharmaceutical Statistics, 4, 287-291. https://doi. org/10.1002/pst. 185

Lobene, R. R., Weatherford, T., Ross, N. M., Lamm, R. A., \& Menaker, L. (1986). A modified gingival index for use in clinical trials. Clinical Preventive Dentistry, 8, 3-6.

McLeroy, K. R., Bibeau, D., Steckler, A., \& Glanz, K. (1988). An ecological perspective on health promotion programs. Health Education Quarterly, 15, 351-377.

Michie, S., Atkins, L., \& West, R. (2014). The behaviour change wheel: A guide to designing interventions. London: Silverback.

Nixon, C. A., Moore, H. J., Douthwaite, W., Gibson, E. L., Vogele, C., \& Kreichauf, S. (2012). Identifying effective behavioural models and behaviour change strategies underpinning preschool- and school-based obesity prevention interventions aimed at 4-6year-olds: A systematic review. Obesity Reviews, 13, 106-117. https://doi.org/10.1111/j.1467-789x.2011.00962.x

O’Leary, T. J., Drake, R. B., \& Naylor, J. E. (1972). The plaque control record. Journal of Periodontology, 43, 38. https://doi. org/10.1902/jop.1972.43.1.38

Parapanisiou, V., Gizani, S., Makou, M., \& Papagiannoulis, L. (2009). Oral health status and behaviour of Greek patients with cleft lip and palate. European Archives of Paediatric Dentistry, $10,85-89$.

Pendry, L., Lashkari, G., \& Bewley, H. (2004). 2003 Children's Dental Health Survey. London: Office for National Statistics.

Vallgarda, S., Nielsen, M. E. J., Hartlev, M., \& Sandoe, P. (2015). Backward- and forward-looking responsibility for obesity: Policies from WHO, the EU and England. European Journal of Public Health, 25, 845-848. https://doi.org/10.1093/eurpub/ ckv076

Wieber, F., von Suchodoletz, A., Heikamp, T., Trommsdorff, G., \& Gollwitzer, P. M. (2011). If-then planning helps school-aged children to ignore attractive distractions. Social Psychology, 42, 39-47. https://doi.org/10.1027/1864-9335/a000041

Publisher's Note Springer Nature remains neutral with regard to jurisdictional claims in published maps and institutional affiliations. 